\title{
A Further Study of the Cytotoxic Consitituents of a Milnamide Producing Sponge
}

Rachel N. Sonnenschein, ${ }^{\dagger}$ Jarrett J. Farias, ${ }^{\dagger}$ Karen Tenney, ${ }^{\dagger}$ Susan L. Mooberry, ${ }^{\ddagger}$ Emil Lobkovsky, ${ }^{\S}$ Jon Clardy, ${ }^{\perp}$ and Phillip Crews ${ }^{\dagger}, *$

${ }^{\dagger}$ Department of Chemistry and Biochemistry \& Institute of Marine Sciences, University of California, Santa Cruz, CA 95064

${ }^{\ddagger}$ Department of Physiology and Medicine, Southwest Foundation for Biomedical Research, San Antonio, TX 78245

${ }^{\S}$ Department of Chemistry and Chemical Biology, Cornell University, Ithaca, New York 14853

${ }^{\perp}$ Department of Biological Chemistry and Molecular Pharmacology, Harvard Medical School, Boston, MA 02115

Document $=$ miln $-2003 \_S I \_R 1 \_3 . d o c(12 / 14 / 03)$

RECEIVED DATE (to be automatically inserted)

\section{[Supporting Information]}

\section{Experimental Procedure.}

Figure S1. ${ }^{1} \mathrm{H}$ NMR spectrum of 2 in $\mathrm{CDCl}_{3}$ at $250 \mathrm{MHz}$.

Figure S2. ${ }^{13} \mathrm{C}$ NMR spectrum of 2 in $\mathrm{CDCl}_{3}$ at $62.5 \mathrm{MHz}$.

Figure S3. ${ }^{1} \mathrm{H}$ NMR spectrum of 3 in $\mathrm{MeOH}-d_{4}$ at $500 \mathrm{MHz}$.

Figure S4. ${ }^{13} \mathrm{C}$ NMR spectrum of 3 in $\mathrm{MeOH}-d_{4}$ at $125 \mathrm{MHz}$.

Table S1. Crystal data and structure refinement for 3.

Table S2. Atomic coordinates $\left(\times 10^{4}\right)$ and equivalent isotropic displacement parameters $\left(\AA^{2} \times 10^{3}\right)$ for 3 .

Table S3. Bond lengths $[\AA]$ and angles $\left[{ }^{\circ}\right]$ for 3 .

Table S4. Anisotropic displacement parameters $\left(\AA^{2} \times 10^{3}\right)$ for 3.

Table S5. Hydrogen coordinates $\left(\times 10^{4}\right)$ and isotropic displacement parameters $\left(\AA^{2} \times 10^{3}\right)$ for 3 .

\footnotetext{
*To whom correspondence should be addressed.
}

Tel.: (831) 459-2603. Fax: (831)-459-2935. E-mail: phil@ chemistry.ucsc.edu 


\section{Experimental Procedures:}

General Experimental Procedures. NMR spectra were recorded at $250 \mathrm{MHz}$ or $500 \mathrm{MHz}$ for ${ }^{1} \mathrm{H}$ NMR and at $62.5 \mathrm{MHz}$ or $125 \mathrm{MHz}$ for ${ }^{13} \mathrm{C}$ NMR. LCMS analysis was performed using an analytical $5 \mu \mathrm{m} \mathrm{C}_{18}$ ODS column with a photodiode array (PDA) detector along with an evaporative light scattering (ELS) detector for compound detection with an electrospray ionization time-of-flight (ESITOF) mass spectrometer for mass detection. Size exclusion chromatography was performed on the crude fractions with Sephadex® LH-20. Flash chromatography was done using silica gel (230-400 mesh). Preparative HPLC was performed with a $6 \mu \mathrm{m} \mathrm{C}_{18}$ ODS column using a single wavelength $(\lambda=254 \mathrm{~nm})$ detector along with an ELS detector. Semi-preparative HPLC was performed with a $5 \mu \mathrm{m} \mathrm{C}_{18}$ ODS column or a $10 \mu \mathrm{m}$ silica normal phase column using a single wavelength $(\lambda=254 \mathrm{~nm})$ for compound detection. Some extraction work was done using an automated accelerated solvent extractor (ASE) system. High resolution MS was obtained using both FAB and ESITOF mass spectrometers. Optical rotation was obtained using a digital polarimeter. UV/vis data was obtained with a monochronometer or PDA detector.

Biological Material, Collection and Identification. Both specimens were collected using SCUBA in Papua New Guinea. Specimen 1 (coll. no. 93100) was collected in 1993 and specimen 2 (coll. no. 99123) was collected in 1999. Preliminary taxonomy was performed in the field by our lab and Ms. M. Sanders. Complete taxonomic identification of specimen 1 was performed by Dr. M. C. Diaz. Complete taxonomic identification of specimen 2 was done by Dr. R. van Soest. Specimen 1 (coll. no. 93100) (167 g dry weight) was collected at depths of 10-20 m in Milne Bay, Papua New Guinea (coordinates available upon request). Specimen 2 (coll. no. 99123) (3.4 kg wet weight), was collected at 8 sites at depths of 20-30 m in the East Fields and Port Moresby regions of Papua New Guinea (S 10 02.6', E $145^{\circ} 28.7^{\prime}$ other GPS coordinates available upon request) and was identified as Auletta sp. 1 by Dr. R. van Soest. Voucher specimens and underwater photos are available (from R.v. S. (voucher of coll. no. 99123) and P.C. (voucher of coll. no. 93100 and UW photos of both)). A voucher of specimen 2 (coll. no. 99123) is being kept in the collections of the Zoological Museum of Amsterdam under registration number ZMAPOR17514.

Extraction and Isolation. Samples were preserved in the field according to our standard laboratory procedures ${ }^{1}$ and stored in a cold room until extraction was performed. Specimen 1 (coll. no. 93100) and part of specimen 2 (approx. 1/5 of wet weight) were extracted 3-5× (until 
supernatant was colorless) with methanol and then the resultant oil was partitioned using a modified Kupchen-type solvent partition scheme as described previously. ${ }^{2}$

Specimen 1 (coll. no. 93100) was first subjected to size-exclusion chromatography using Sephadex ${ }^{\circledR} \mathrm{LH}-20$ (7:3 MeOH: $\left.\mathrm{CH}_{2} \mathrm{Cl}_{2}\right)$ to give 8 fractions. Fraction 4 (145.5 mg) was then subjected to silica gel flash chromatography (75:25 EtOAc:hexanes) to yield three fractions. Fraction 1 (101.5 mg) was further purified using normal phase semi-preparative HPLC (10:90 hexanes:EtOAc) to yield $\mathbf{1}(26 \mathrm{mg}), \mathbf{2}(9 \mathrm{mg}), \mathbf{3}(4 \mathrm{mg})$ and $\mathbf{5}(38 \mathrm{mg})$. This sample of $\mathbf{3}$ decomposed on standing.

A portion of specimen 2 (coll. no. 99123) (approx. 1/5 of wet weight) was first subjected to size-exclusion chromatography using Sephadex® $\mathrm{LH}-20\left(7: 3 \mathrm{MeOH}: \mathrm{CH}_{2} \mathrm{Cl}_{2}\right)$ to give 9 fractions. Based on bioactivity and LRMS data fractions 5 and 6 were further fractionated using reversed-phase HPLC. The $5^{\text {th }}$ Sephadex $®$ fraction $(52.9 \mathrm{mg})$ was fractioned using reversed-phase semi-preparative $\mathrm{HPLC}\left(50\right.$ to $100 \% \mathrm{ACN}$ in $\mathrm{H}_{2} \mathrm{O}$ ) to afford mostly pure milnamide C (3) (fraction $\mathrm{H} 8,2.5 \mathrm{mg}$ ) as well as a pure $6(2 \mathrm{mg}$ ) and impure $9(2.0 \mathrm{mg})$. Fraction $\mathrm{H} 8$ was further purified using isocratic reversed-phase semi-preparative HPLC (58:42 ACN: $\left.\mathrm{H}_{2} 0\right)$ to yield 6 fractions. Fraction $5(1.0 \mathrm{mg})$ appeared to contain a mixture of milnamide $\mathrm{C}(3)$ and a compound of $568 \mathrm{MW}$ compound. The $6^{\text {th }}$ Sephadex® fraction $(72 \mathrm{mg}$ ) was fractionated using reversed-phase semi-preparative $\mathrm{HPLC}\left(50\right.$ to $100 \% \mathrm{ACN}$ in $\mathrm{H}_{2} \mathrm{O}$ ) to give $9(1.6 \mathrm{mg}$ ) and a mixture of 6 and 7 (2.8 $\mathrm{mg})$.

Another portion of specimen 2 (coll. no. 99123) (approx. 1/5 of wet weight) was extracted using the ASE to give three fractions. The procedure for extraction with the ASE is as follows. The methanol that the sponge was stored in was evaporated and the resultant oil was applied to the wet sponge, which was then allowed to air dry. After this the sponge was ground up and then partitioned using the ASE by first extracting with hexanes $(3 \times)$, then with $\mathrm{CH}_{2} \mathrm{Cl}_{2}(3 \times)$ and finally with $\mathrm{MeOH}(3 \times)$. The $\mathrm{CH}_{2} \mathrm{Cl}_{2}$ fraction (XFD) was subjected to preparative reversed phase HPLC (10 to $100 \% \mathrm{ACN}$ in $\mathrm{H}_{2} \mathrm{O}$ ) to yield 20 fractions. Fractions 8 and 9 were fractionated using reversed-phase semi-preparative HPLC (50 to 70\% $\mathrm{ACN}$ in $\mathrm{H}_{2} \mathrm{O}$ ) to yield $8(2 \mathrm{mg})$ and 7 (2 $\left.\mathrm{mg}\right)$, respectively. Various fractions from the preparative HPLC shown to contain $\mathbf{3}$ were combined (17.3 mg) and fractionated using reversed-phase semi-preparative $\mathrm{HPLC}$ ( 55 to $80 \% \mathrm{ACN}$ in $\mathrm{H}_{2} \mathrm{O}$ ) to yield 8 fractions. Fraction 6 (3.4 mg) was further purified using reversed-phase semi-preparative HPLC (58 to $63 \% \mathrm{ACN}$ in $\mathrm{H}_{2} \mathrm{O}$ ) to yield 6 fractions. Fraction $2(0.7 \mathrm{mg})$ contained a compound of $\mathrm{MW}=568$ and fraction $4(2.4 \mathrm{mg})$ appeared to contain a mixture of compounds including $\mathbf{3}$. This 
fraction was further purified using isocratic reversed-phase semi-preparative HPLC (58:42 $\left.\mathrm{ACN}: \mathrm{H}_{2} 0\right)$ to give 6 fractions. Fraction $3(1 \mathrm{mg})$ appeared to contain the same compound $(M W=568)$ as the previous 2nd fraction (from Sephadex fraction 6), attempts to obtain NMR data on these two fractions proved fruitless. Fraction $5(1.0 \mathrm{mg})$ appeared to contain a mixture of milnamide C (3) and the $568 \mathrm{MW}$ compound. Based on NMR data this fraction 5 was combined with the fraction from the first extraction of this collection that contained $\mathbf{3}$ and a compound of $\mathrm{MW}=568$ to give a fraction of $2.0 \mathrm{mg}$, however, when NMR was run on this fraction 3 appeared to have degraded into the compound with a MW of 568. Attempts to obtain NMR data on this fraction proved fruitless.

Another portion of specimen 1 which had been extracted using our modified kapchun type extraction scheme as previously described ${ }^{2}$ was subjected to size-exclusion chromatography as above to give 9 fractions. Fraction $4(70.0 \mathrm{mg})$ was fractioned using reversed-phase semi-preparative HPLC (50 to $100 \% \mathrm{ACN}$ in $\mathrm{H}_{2} \mathrm{O}$ ) to yield pure 3 (4.0 mg).

All known compounds purified were identified by comparison to literature values.

Milnamide B (2). Yellow solid; $[\alpha]_{\mathrm{D}}^{27}-90^{\circ}\left(c 0.08, \mathrm{CH}_{2} \mathrm{Cl}_{2}\right)$; UV(MeOH) $\lambda_{\max }(\varepsilon) 223$ (13950), 281 (2795), 294 (2905); HRFABMS $m / z, 527.3601[\mathrm{M}+\mathrm{H}]^{+}(\Delta-0.4 \mathrm{mmu}$ of calcd. for $\mathrm{C}_{30} \mathrm{H}_{46} \mathrm{~N}_{4} \mathrm{O}_{4}$ ); NMR data in accordance with literature values.

Milnamide C (3). Yellow crystals; $[\alpha]_{\mathrm{D}}^{28}+68^{\circ}(c 0.06, \mathrm{MeOH}) ; \mathrm{UV}(\mathrm{MeOH}) \lambda_{\max }(\varepsilon) 210$ (19551), 225 (18445), 308 (7461); HRESITOFMS m/z $553.3335[\mathrm{M}+\mathrm{H}]^{+}(\Delta-5.0 \mathrm{mmu}$ of calcd. for $\mathrm{C}_{31} \mathrm{H}_{45} \mathrm{~N}_{4} \mathrm{O}_{5}$ ); see Table 1 for ${ }^{1} \mathrm{H}$ and ${ }^{13} \mathrm{C}$ NMR. The single-crystal $\mathrm{X}$-ray analysis was conducted as follows.

X-ray crystallography of 3. Suitable crystals were obtained from 67:33 ACN:H $\mathrm{H}_{2} \mathrm{O}$ by the vapor diffusion method. This crystal $\left(0.30 \times 0.15 \times 0.10 \mathrm{~mm}^{3}\right)$ was mounted on a Bruker SMART diffractometer $\left(\mathrm{MoK}_{\alpha} ;-133^{\circ} \mathrm{C}\right)$. A hemisphere of data was taken using a narrow-scan routine (1818 frames, $0.3^{\circ}$ steps $\omega$-scan, exposure time was $30 \mathrm{~s} /$ frame, $2 \theta_{\max }=53.16^{\circ}$ ). Raw data was integrated with the Bruker SAINT+ program ${ }^{3}$ to yield a total 20798 reflections, of which 6349 were independent $\left(\mathrm{R}_{\mathrm{int}}=8.39 \%\right.$, completeness $\left.99.7 \%\right)$ and 5051 with $\mathrm{I}>2 \sigma(\mathrm{I})$. Data was corrected for absorption using SADABS program (min. and max. transmission are 0.9763 and 0.9920 , respectively). ${ }^{4}$ The structure was solved by direct methods and refined by full matrix least square on $\mathrm{F}^{2}$ techniques using anisotropic displacement parameters for all non-hydrogen atoms. ${ }^{5}$ All hydrogen atoms were found in the difference Fourier map and refined isotropically. At final convergence, $\mathrm{R}_{1}=4.84 \%$ and $\mathrm{GOF}=1.077$ for 537 parameters. Additional information about 
these data includes crystal data and structure refinement in Table S1, atomic coordinates in Table S2, bond length and angles in Table S3, anisotropic displacement parameters in Table S4 and hydrogen coordinates in Table S5.

Biological Activity. Compound induced changes in microtubules were evaluated by immunofluorescence techniques as previously described. ${ }^{6}$ Changes in microfilaments were evaluated using a phalloidin assay as previously described. ${ }^{7}$ The antiproliferative activities of the compounds were evaluated using the sulforhodamine B assay as previously described. ${ }^{6}$ 


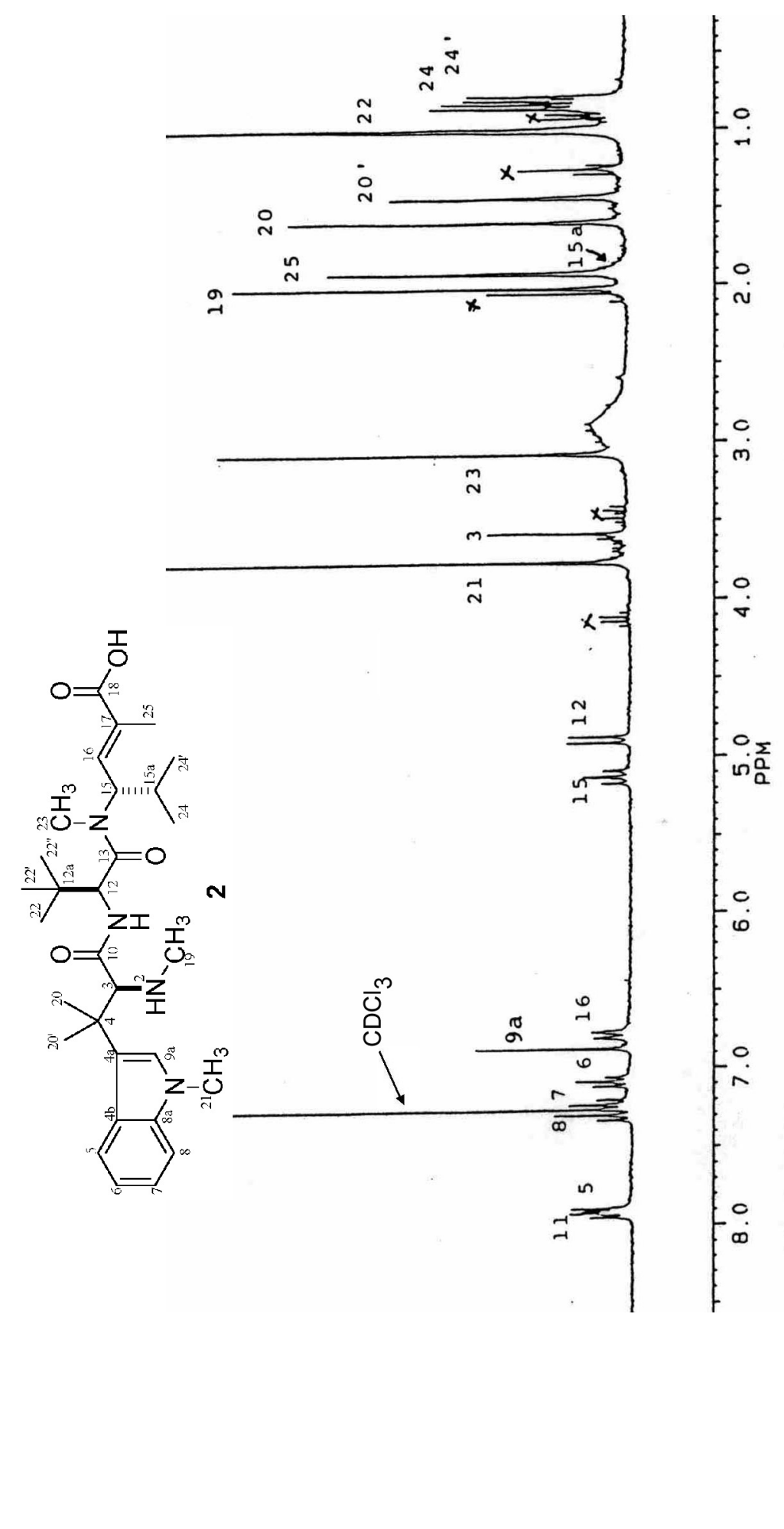


v

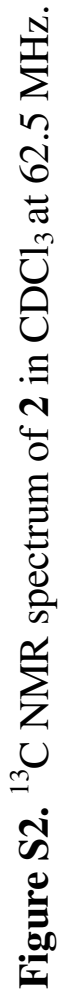

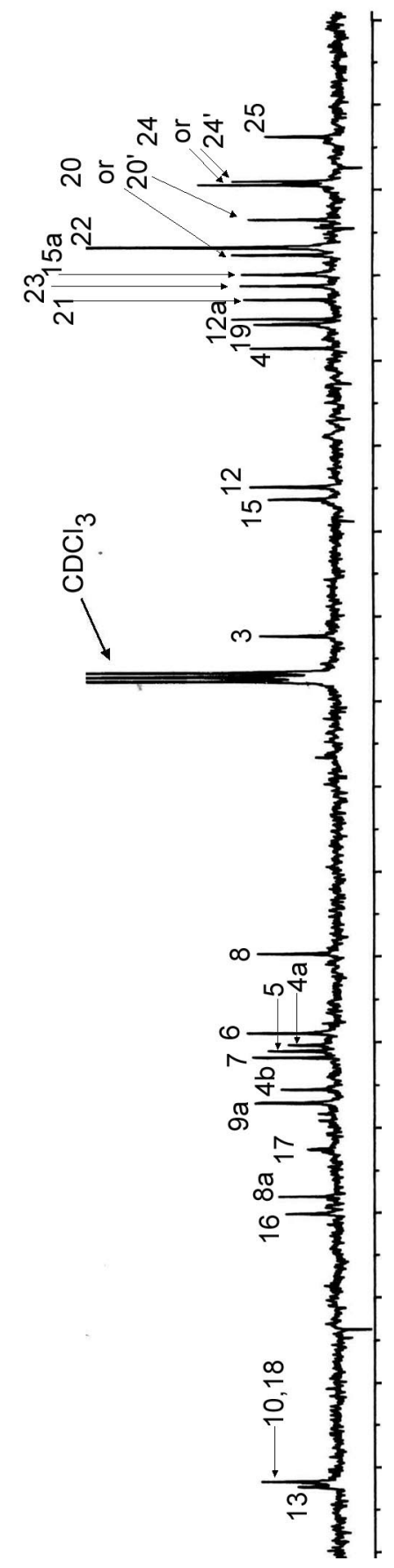




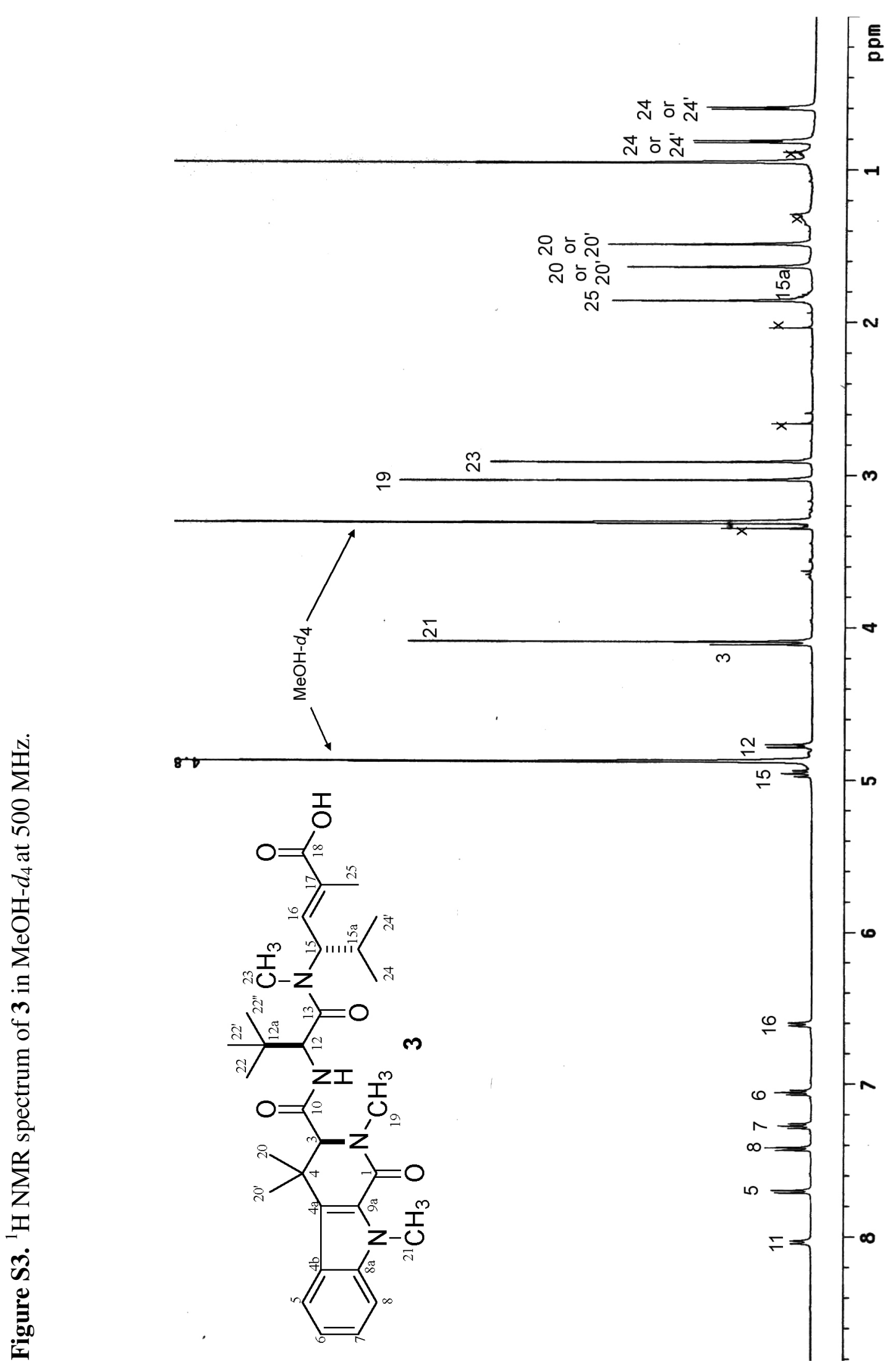



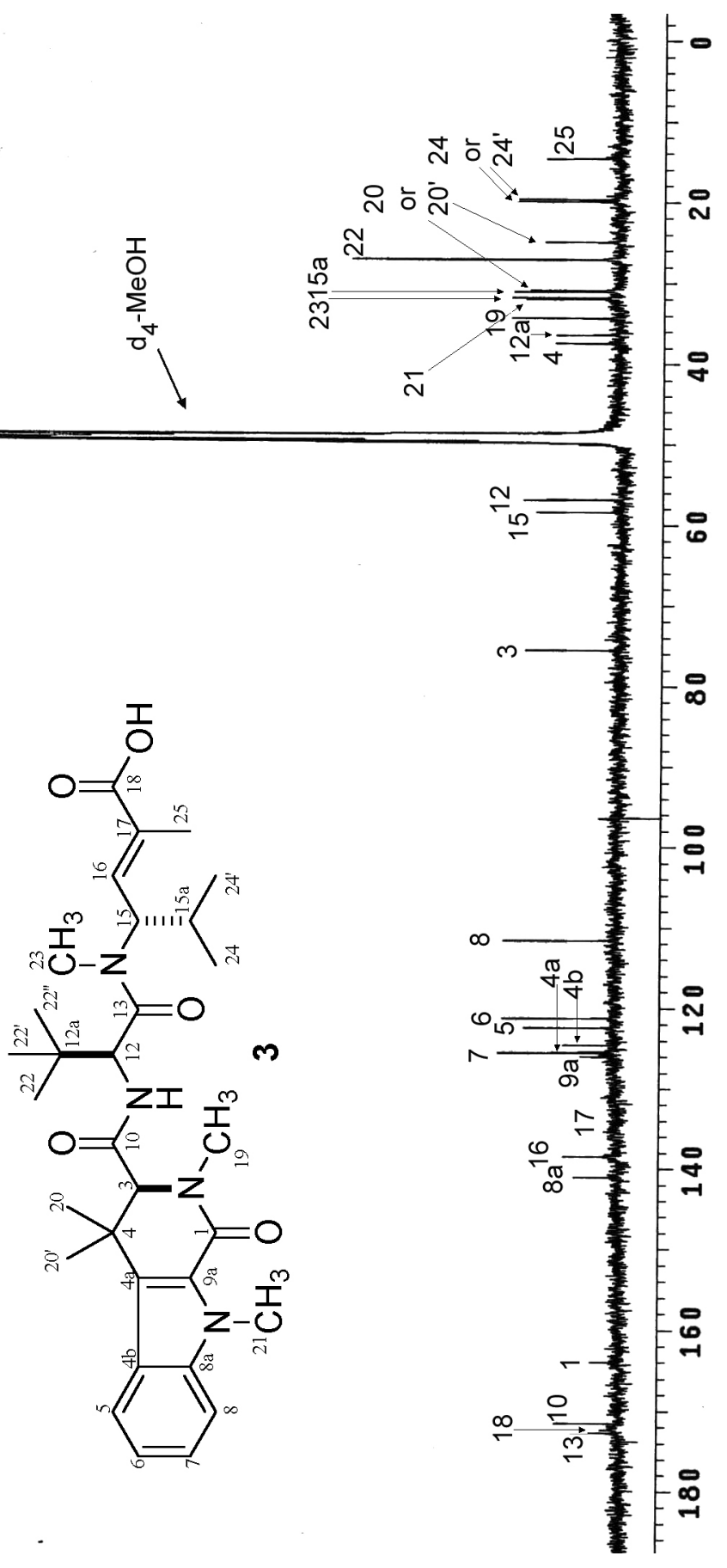
Table S1. Crystal data and structure refinement for $\mathbf{3}$.

Identification code

Empirical formula

Formula weight

Temperature

Wavelength

Crystal system

Space group

Unit cell dimensions

Volume

Z

Density (calculated)

Absorption coefficient

$\mathrm{F}(000)$

Crystal size

Theta range for data collection

Index ranges

Reflections collected

Independent reflections

Completeness to theta $=26.38^{\circ}$

Absorption correction

Max. and min. transmission

Refinement method

Data / restraints / parameters

Goodness-of-fit on $\mathrm{F}^{2}$

Final $\mathrm{R}$ indices [I $>2 \operatorname{sigma}(\mathrm{I})]$

$\mathrm{R}$ indices (all data)

Absolute structure parameter

Largest diff. peak and hole milnamide $\mathrm{C}$ (pc487)

C31 H44 N4 O5

552.70

$-140(2) \mathrm{K}$

$0.71073 \AA$

Orthorhombic

P2(1)2(1)2(1)

$\mathrm{a}=13.0924(8) \AA$

$\alpha=90^{\circ}$.

$\mathrm{b}=15.0715(9) \AA \quad \beta=90^{\circ}$.

$\mathrm{c}=15.7587(9) \AA \quad \gamma=90^{\circ}$.

3109.5(3) $\AA^{3}$

4

$1.181 \mathrm{Mg} / \mathrm{m}^{3}$

$0.080 \mathrm{~mm}^{-1}$

1192

$0.30 \times 0.15 \times 0.10 \mathrm{~mm}^{3}$

1.87 to $26.38^{\circ}$.

$-16<=\mathrm{h}<=14,-18<=\mathrm{k}<=18,-19<=\mathrm{l}<=19$

20798

$6349[\mathrm{R}(\mathrm{int})=0.0839]$

$99.7 \%$

SADABS

0.9920 and 0.9763

Full-matrix least-squares on $\mathrm{F}^{2}$

6349 / 0 / 537

1.077

$\mathrm{R} 1=0.0484, \mathrm{wR} 2=0.0942$

$\mathrm{R} 1=0.0713, \mathrm{wR} 2=0.1027$

$0.8(11)$

0.180 and -0.207 e. $\AA^{-3}$ 
Table S2. Atomic coordinates $\left(\times 10^{4}\right)$ and equivalent isotropic displacement parameters $\left(\AA^{2} \times 10^{3}\right)$ for 3. $U(e q)$ is defined as one third of the trace of the orthogonalized $U^{i j}$ tensor.

\begin{tabular}{|c|c|c|c|c|}
\hline & $\mathrm{x}$ & $\mathrm{y}$ & $\mathrm{z}$ & $\mathrm{U}(\mathrm{eq})$ \\
\hline $\mathrm{O}(1)$ & 10831(1) & $5267(1)$ & $-529(1)$ & $33(1)$ \\
\hline $\mathrm{O}(2)$ & $8790(1)$ & $3643(1)$ & $812(1)$ & $27(1)$ \\
\hline $\mathrm{O}(3)$ & 6113(1) & $3607(1)$ & 2092(1) & $21(1)$ \\
\hline $\mathrm{O}(4)$ & $4962(1)$ & $80(1)$ & $3016(1)$ & $29(1)$ \\
\hline $\mathrm{O}(5)$ & $4048(1)$ & $614(1)$ & 4103(1) & $33(1)$ \\
\hline $\mathrm{C}(1)$ & $10238(2)$ & $5242(2)$ & $74(2)$ & $22(1)$ \\
\hline $\mathrm{N}(2)$ & $9207(1)$ & $5347(1)$ & $-33(1)$ & $21(1)$ \\
\hline $\mathrm{C}(3)$ & $8464(2)$ & $5211(2)$ & $653(2)$ & $20(1)$ \\
\hline $\mathrm{C}(4 \mathrm{~A})$ & $9928(2)$ & $5210(1)$ & $1648(2)$ & $20(1)$ \\
\hline $\mathrm{C}(4 \mathrm{~B})$ & 10499(2) & $4950(1)$ & $2381(2)$ & $22(1)$ \\
\hline $\mathrm{C}(4)$ & $8848(2)$ & $5555(1)$ & $1522(2)$ & $21(1)$ \\
\hline $\mathrm{C}(5)$ & $10310(2)$ & $4881(2)$ & $3257(2)$ & $26(1)$ \\
\hline$C(6)$ & $11059(2)$ & $4562(2)$ & $3782(2)$ & $32(1)$ \\
\hline $\mathrm{C}(7)$ & $12021(2)$ & $4312(2)$ & $3468(2)$ & $32(1)$ \\
\hline $\mathrm{C}(8 \mathrm{~A})$ & $11472(2)$ & $4692(1)$ & $2080(2)$ & $22(1)$ \\
\hline $\mathrm{C}(8)$ & $12241(2)$ & $4375(2)$ & $2618(2)$ & $28(1)$ \\
\hline $\mathrm{N}(9)$ & $11504(1)$ & 4799(1) & 1211(1) & $22(1)$ \\
\hline $\mathrm{C}(9 \mathrm{~A})$ & $10552(2)$ & $5106(1)$ & $959(2)$ & $20(1)$ \\
\hline$C(10)$ & $8157(2)$ & $4225(1)$ & $692(1)$ & $18(1)$ \\
\hline $\mathrm{N}(11)$ & $7159(1)$ & $4059(1)$ & $573(1)$ & $18(1)$ \\
\hline $\mathrm{C}(12)$ & $6790(2)$ & $3162(1)$ & $742(2)$ & 19(1) \\
\hline$C(12 A)$ & $5804(2)$ & $2905(2)$ & $250(2)$ & $26(1)$ \\
\hline $\mathrm{C}(13)$ & $6661(2)$ & $3068(1)$ & $1706(2)$ & $17(1)$ \\
\hline $\mathrm{N}(14)$ & $7163(1)$ & 2413(1) & $2107(1)$ & $17(1)$ \\
\hline$C(15 A)$ & $7947(2)$ & $2165(2)$ & $3547(2)$ & $22(1)$ \\
\hline$C(15)$ & $6961(2)$ & $2285(1)$ & $3022(1)$ & $16(1)$ \\
\hline$C(16)$ & $6231(2)$ & $1515(1)$ & $3107(1)$ & $18(1)$ \\
\hline $\mathrm{C}(17)$ & $5356(2)$ & $1518(2)$ & $3534(2)$ & $23(1)$ \\
\hline $\mathrm{C}(18)$ & $4727(2)$ & $697(2)$ & $3578(2)$ & $24(1)$ \\
\hline $\mathrm{C}(19)$ & $8809(2)$ & $5305(2)$ & $-897(2)$ & $28(1)$ \\
\hline $\mathrm{C}\left(20^{\prime}\right)$ & $8116(2)$ & $5246(2)$ & $2222(2)$ & $25(1)$ \\
\hline $\mathrm{C}(20)$ & $8852(2)$ & $6579(2)$ & $1503(2)$ & $31(1)$ \\
\hline $\mathrm{C}(21)$ & $12394(2)$ & 4590(2) & $693(2)$ & $29(1)$ \\
\hline C(22") & $6068(3)$ & $2847(2)$ & $-694(2)$ & $38(1)$ \\
\hline$C\left(22^{\prime}\right)$ & $5461(3)$ & 1992(2) & $567(2)$ & $43(1)$ \\
\hline $\mathrm{C}(22)$ & 4929(2) & $3565(2)$ & $366(2)$ & $37(1)$ \\
\hline $\mathrm{C}(23)$ & 7821(2) & 1762(2) & 1674(2) & $27(1)$ \\
\hline$C\left(24^{\prime}\right)$ & $8682(2)$ & 2934(2) & $3370(2)$ & $29(1)$ \\
\hline$C(24)$ & $7683(2)$ & 2122(2) & $4490(2)$ & $32(1)$ \\
\hline $\mathrm{C}(25)$ & 4941(3) & $2285(2)$ & $4035(3)$ & $46(1)$ \\
\hline
\end{tabular}


Table S3. Bond lengths $[\AA]$ and angles $\left[^{\circ}\right]$ for $\mathbf{3}$.

\begin{tabular}{|c|c|}
\hline $\mathrm{O}(1)-\mathrm{C}(1)$ & $1.227(3)$ \\
\hline $\mathrm{O}(2)-\mathrm{C}(10)$ & $1.221(3)$ \\
\hline $\mathrm{O}(3)-\mathrm{C}(13)$ & $1.243(3)$ \\
\hline $\mathrm{O}(4)-\mathrm{C}(18)$ & $1.320(3)$ \\
\hline $\mathrm{O}(5)-\mathrm{C}(18)$ & $1.221(3)$ \\
\hline $\mathrm{C}(1)-\mathrm{N}(2)$ & $1.369(3)$ \\
\hline $\mathrm{C}(1)-\mathrm{C}(9 \mathrm{~A})$ & $1.469(3)$ \\
\hline N(2)-C(19) & $1.459(3)$ \\
\hline $\mathrm{N}(2)-\mathrm{C}(3)$ & $1.469(3)$ \\
\hline C(3)-C(10) & $1.541(3)$ \\
\hline$C(3)-C(4)$ & $1.548(3)$ \\
\hline $\mathrm{C}(4 \mathrm{~A})-\mathrm{C}(9 \mathrm{~A})$ & $1.368(3)$ \\
\hline$C(4 A)-C(4 B)$ & $1.431(3)$ \\
\hline $\mathrm{C}(4 \mathrm{~A})-\mathrm{C}(4)$ & $1.520(3)$ \\
\hline $\mathrm{C}(4 \mathrm{~B})-\mathrm{C}(5)$ & $1.405(3)$ \\
\hline $\mathrm{C}(4 \mathrm{~B})-\mathrm{C}(8 \mathrm{~A})$ & $1.414(3)$ \\
\hline $\mathrm{C}(4)-\mathrm{C}\left(20^{\prime}\right)$ & $1.533(3)$ \\
\hline$C(4)-C(20)$ & $1.543(3)$ \\
\hline$C(5)-C(6)$ & $1.371(4)$ \\
\hline$C(6)-C(7)$ & $1.405(4)$ \\
\hline $\mathrm{C}(7)-\mathrm{C}(8)$ & $1.372(4)$ \\
\hline $\mathrm{C}(8 \mathrm{~A})-\mathrm{N}(9)$ & $1.379(3)$ \\
\hline $\mathrm{C}(8 \mathrm{~A})-\mathrm{C}(8)$ & $1.401(3)$ \\
\hline $\mathrm{N}(9)-\mathrm{C}(9 \mathrm{~A})$ & $1.387(3)$ \\
\hline $\mathrm{N}(9)-\mathrm{C}(21)$ & $1.458(3)$ \\
\hline $\mathrm{C}(10)-\mathrm{N}(11)$ & $1.343(3)$ \\
\hline $\mathrm{N}(11)-\mathrm{C}(12)$ & $1.461(3)$ \\
\hline $\mathrm{C}(12)-\mathrm{C}(13)$ & $1.534(3)$ \\
\hline$C(12)-C(12 A)$ & $1.555(3)$ \\
\hline$C(12 A)-C(22)$ & $1.528(4)$ \\
\hline $\mathrm{C}(12 \mathrm{~A})-\mathrm{C}\left(22^{\prime \prime}\right)$ & $1.530(4)$ \\
\hline $\mathrm{C}(12 \mathrm{~A})-\mathrm{C}\left(22^{\prime}\right)$ & $1.531(4)$ \\
\hline $\mathrm{C}(13)-\mathrm{N}(14)$ & $1.344(3)$ \\
\hline $\mathrm{N}(14)-\mathrm{C}(23)$ & $1.474(3)$ \\
\hline N(14)-C(15) & $1.479(3)$ \\
\hline $\mathrm{C}(15 \mathrm{~A})-\mathrm{C}(24)$ & $1.527(4)$ \\
\hline $\mathrm{C}(15 \mathrm{~A})-\mathrm{C}\left(24^{\prime}\right)$ & $1.532(3)$ \\
\hline$C(15 A)-C(15)$ & $1.543(3)$ \\
\hline$C(15)-C(16)$ & $1.508(3)$ \\
\hline$C(16)-C(17)$ & $1.328(3)$ \\
\hline C(17)-C(18) & $1.489(3)$ \\
\hline $\mathrm{C}(17)-\mathrm{C}(25)$ & $1.501(4)$ \\
\hline $\mathrm{O}(1)-\mathrm{C}(1)-\mathrm{N}(2)$ & $121.7(2)$ \\
\hline $\mathrm{O}(1)-\mathrm{C}(1)-\mathrm{C}(9 \mathrm{~A})$ & $124.2(2)$ \\
\hline $\mathrm{N}(2)-\mathrm{C}(1)-\mathrm{C}(9 \mathrm{~A})$ & $114.2(2)$ \\
\hline $\mathrm{C}(1)-\mathrm{N}(2)-\mathrm{C}(19)$ & $117.5(2)$ \\
\hline $\mathrm{C}(1)-\mathrm{N}(2)-\mathrm{C}(3)$ & 123.11(19) \\
\hline $\mathrm{C}(19)-\mathrm{N}(2)-\mathrm{C}(3)$ & 116.38(19) \\
\hline $\mathrm{N}(2)-\mathrm{C}(3)-\mathrm{C}(10)$ & $109.62(18)$ \\
\hline $\mathrm{N}(2)-\mathrm{C}(3)-\mathrm{C}(4)$ & 112.91(18) \\
\hline $\mathrm{C}(10)-\mathrm{C}(3)-\mathrm{C}(4)$ & 111.91(18) \\
\hline $\mathrm{C}(9 \mathrm{~A})-\mathrm{C}(4 \mathrm{~A})-\mathrm{C}(4 \mathrm{~B})$ & $107.30(19)$ \\
\hline
\end{tabular}




$\begin{array}{ll}\mathrm{C}(9 \mathrm{~A})-\mathrm{C}(4 \mathrm{~A})-\mathrm{C}(4) & 119.5(2) \\ \mathrm{C}(4 \mathrm{~B})-\mathrm{C}(4 \mathrm{~A})-\mathrm{C}(4) & 133.2(2) \\ \mathrm{C}(5)-\mathrm{C}(4 \mathrm{~B})-\mathrm{C}(8 \mathrm{~A}) & 117.9(2) \\ \mathrm{C}(5)-\mathrm{C}(4 \mathrm{~B})-\mathrm{C}(4 \mathrm{~A}) & 136.2(2) \\ \mathrm{C}(8 \mathrm{~A})-\mathrm{C}(4 \mathrm{~B})-\mathrm{C}(4 \mathrm{~A}) & 105.9(2) \\ \mathrm{C}(4 \mathrm{~A})-\mathrm{C}(4)-\mathrm{C}\left(20^{\prime}\right) & 112.6(2) \\ \mathrm{C}(4 \mathrm{~A})-\mathrm{C}(4)-\mathrm{C}(20) & 110.04(19) \\ \mathrm{C}\left(20^{\prime}\right)-\mathrm{C}(4)-\mathrm{C}(20) & 108.7(2) \\ \mathrm{C}(4 \mathrm{~A})-\mathrm{C}(4)-\mathrm{C}(3) & 107.62(18) \\ \mathrm{C}\left(20^{\prime}\right)-\mathrm{C}(4)-\mathrm{C}(3) & 109.31(18) \\ \mathrm{C}(20)-\mathrm{C}(4)-\mathrm{C}(3) & 108.6(2) \\ \mathrm{C}(6)-\mathrm{C}(5)-\mathrm{C}(4 \mathrm{~B}) & 119.6(2) \\ \mathrm{C}(5)-\mathrm{C}(6)-\mathrm{C}(7) & 121.5(3) \\ \mathrm{C}(8)-\mathrm{C}(7)-\mathrm{C}(6) & 120.9(3) \\ \mathrm{N}(9)-\mathrm{C}(8 \mathrm{~A})-\mathrm{C}(8) & 128.2(2) \\ \mathrm{N}(9)-\mathrm{C}(8 \mathrm{~A})-\mathrm{C}(4 \mathrm{~B}) & 109.2(2) \\ \mathrm{C}(8)-\mathrm{C}(8 \mathrm{~A})-\mathrm{C}(4 \mathrm{~B}) & 122.6(2) \\ \mathrm{C}(7)-\mathrm{C}(8)-\mathrm{C}(8 \mathrm{~A}) & 117.6(2) \\ \mathrm{C}(8 \mathrm{~A})-\mathrm{N}(9)-\mathrm{C}(9 \mathrm{~A}) & 107.22(18) \\ \mathrm{C}(8 \mathrm{~A})-\mathrm{N}(9)-\mathrm{C}(21) & 123.8(2) \\ \mathrm{C}(9 \mathrm{~A})-\mathrm{N}(9)-\mathrm{C}(21) & 129.0(2) \\ \mathrm{C}(4 \mathrm{~A})-\mathrm{C}(9 \mathrm{~A})-\mathrm{N}(9) & 110.4(2) \\ \mathrm{C}(4 \mathrm{~A})-\mathrm{C}(9 \mathrm{~A})-\mathrm{C}(1) & 124.7(2) \\ \mathrm{N}(9)-\mathrm{C}(9 \mathrm{~A})-\mathrm{C}(1) & 124.8(2) \\ \mathrm{O}(2)-\mathrm{C}(10)-\mathrm{N}(11) & 123.3(2) \\ \mathrm{O}(2)-\mathrm{C}(10)-\mathrm{C}(3) & 121.47(19) \\ \mathrm{N}(11)-\mathrm{C}(10)-\mathrm{C}(3) & 115.27(19) \\ \mathrm{C}(10)-\mathrm{N}(11)-\mathrm{C}(12) & 117.95(19) \\ \mathrm{N}(11)-\mathrm{C}(12)-\mathrm{C}(13) & 107.56(17) \\ \mathrm{N}(11)-\mathrm{C}(12)-\mathrm{C}(12 \mathrm{~A}) & 114.50(19) \\ \mathrm{C}(13)-\mathrm{C}(12)-\mathrm{C}(12 \mathrm{~A}) & 112.34(18) \\ \mathrm{C}(22)-\mathrm{C}(12 \mathrm{~A})-\mathrm{C}(22 ") & 108.9(2) \\ \mathrm{C}(22)-\mathrm{C}(12 \mathrm{~A})-\mathrm{C}\left(22^{\prime}\right) & 109.0(2) \\ \mathrm{C}\left(22^{\prime}\right)-\mathrm{C}(12 \mathrm{~A})-\mathrm{C}\left(22^{\prime}\right) & 109.4(2) \\ \mathrm{C}(22)-\mathrm{C}(12 \mathrm{~A})-\mathrm{C}(12) & 113.6(2) \\ \left.\mathrm{C}(22)^{\prime \prime}\right)-\mathrm{C}(12 \mathrm{~A})-\mathrm{C}(12) & 108.2(2) \\ \mathrm{C}\left(22^{\prime}\right)-\mathrm{C}(12 \mathrm{~A})-\mathrm{C}(12) & 107.7(2) \\ \mathrm{O}(3)-\mathrm{C}(13)-\mathrm{N}(14) & 122.1(2) \\ \mathrm{O}(3)-\mathrm{C}(13)-\mathrm{C}(12) & 119.22(19) \\ \mathrm{N}(14)-\mathrm{C}(13)-\mathrm{C}(12) & 118.69(19) \\ \mathrm{C}(13)-\mathrm{N}(14)-\mathrm{C}(23) & 123.83(19) \\ \mathrm{C}(13)-\mathrm{N}(14)-\mathrm{C}(15) & 117.90(17) \\ \mathrm{C}(23)-\mathrm{N}(14)-\mathrm{C}(15) & 117.99(18) \\ \mathrm{C}(24)-\mathrm{C}(15 \mathrm{~A})-\mathrm{C}\left(24^{\prime}\right) & 110.6(2) \\ \mathrm{C}(24)-\mathrm{C}(15 \mathrm{~A})-\mathrm{C}(15) & 109.7(2) \\ \left.\mathrm{C}(24)^{\prime}\right)-\mathrm{C}(15 \mathrm{~A})-\mathrm{C}(15) & 109.86(18) \\ \mathrm{N}(14)-\mathrm{C}(15)-\mathrm{C}(16) & 107.45(17) \\ \mathrm{N}(14)-\mathrm{C}(15)-\mathrm{C}(15 \mathrm{~A}) & 112.81(18) \\ \mathrm{C}(16)-\mathrm{C}(15)-\mathrm{C}(15 \mathrm{~A}) & 113.14(17)-\mathrm{C}(17) \\ \mathrm{C}(18)-\mathrm{C}(17)-\mathrm{C}(18)-\mathrm{C}(25) & 126.0(2) \\ \mathrm{O}(5)-\mathrm{C}(18)-\mathrm{O}(4) & 119.9(2) \\ & \end{array}$


$\mathrm{O}(5)-\mathrm{C}(18)-\mathrm{C}(17)$

$121.3(2)$

$\mathrm{O}(4)-\mathrm{C}(18)-\mathrm{C}(17)$

$115.1(2)$

Symmetry transformations used to generate equivalent atoms: 
Table S4. Anisotropic displacement parameters $\left(\AA^{2} \times 10^{3}\right)$ for 3 . The anisotropic displacement factor exponent takes the form: $-2 \pi^{2}\left[h^{2} a^{* 2} U^{11}+\ldots+2 h \mathrm{k} \mathrm{a}^{*} \mathrm{~b}^{*} \mathrm{U}^{12}\right]$.

\begin{tabular}{|c|c|c|c|c|c|c|}
\hline & $\mathrm{U}^{11}$ & $\mathrm{U}^{22}$ & $\mathrm{U}^{33}$ & $\mathrm{U}^{23}$ & $\mathrm{U}^{13}$ & $\mathrm{U}^{12}$ \\
\hline $\mathrm{O}(1)$ & $24(1)$ & $45(1)$ & $29(1)$ & $0(1)$ & $9(1)$ & $-4(1)$ \\
\hline $\mathrm{O}(2)$ & $17(1)$ & 21(1) & $42(1)$ & $2(1)$ & $0(1)$ & $3(1)$ \\
\hline $\mathrm{O}(3)$ & 21(1) & 19(1) & $25(1)$ & $0(1)$ & $2(1)$ & $7(1)$ \\
\hline $\mathrm{O}(4)$ & $32(1)$ & $24(1)$ & $33(1)$ & $-6(1)$ & $7(1)$ & $-15(1)$ \\
\hline $\mathrm{O}(5)$ & $24(1)$ & $31(1)$ & $45(1)$ & $-5(1)$ & $12(1)$ & $-9(1)$ \\
\hline $\mathrm{C}(1)$ & 21(1) & $18(1)$ & $28(1)$ & $-2(1)$ & $4(1)$ & $-3(1)$ \\
\hline $\mathrm{N}(2)$ & $17(1)$ & $23(1)$ & $22(1)$ & $3(1)$ & $2(1)$ & $-1(1)$ \\
\hline C(3) & $13(1)$ & 21(1) & $26(1)$ & $3(1)$ & $4(1)$ & $4(1)$ \\
\hline $\mathrm{C}(4 \mathrm{~A})$ & $17(1)$ & 20(1) & $24(1)$ & $-1(1)$ & $1(1)$ & $-4(1)$ \\
\hline $\mathrm{C}(4 \mathrm{~B})$ & 19(1) & $18(1)$ & $30(1)$ & $-3(1)$ & $-2(1)$ & $-6(1)$ \\
\hline$C(4)$ & $17(1)$ & 21(1) & $25(1)$ & $-2(1)$ & $2(1)$ & $-1(1)$ \\
\hline$C(5)$ & $23(1)$ & $27(1)$ & $30(1)$ & $-3(1)$ & $-1(1)$ & $-5(1)$ \\
\hline$C(6)$ & $31(2)$ & $34(1)$ & $30(2)$ & $3(1)$ & $-6(1)$ & $-10(1)$ \\
\hline$C(7)$ & $28(1)$ & $29(1)$ & $39(2)$ & $3(1)$ & $-12(1)$ & $0(1)$ \\
\hline $\mathrm{C}(8 \mathrm{~A})$ & 19(1) & $17(1)$ & 31(1) & $-2(1)$ & $-4(1)$ & $-4(1)$ \\
\hline C(8) & 19(1) & $26(1)$ & $40(2)$ & $0(1)$ & $-5(1)$ & $-3(1)$ \\
\hline $\mathrm{N}(9)$ & $13(1)$ & $24(1)$ & $30(1)$ & $-1(1)$ & 1(1) & $-1(1)$ \\
\hline $\mathrm{C}(9 \mathrm{~A})$ & $17(1)$ & $18(1)$ & $26(1)$ & $0(1)$ & $0(1)$ & $-3(1)$ \\
\hline $\mathrm{C}(10)$ & $15(1)$ & $22(1)$ & $16(1)$ & $2(1)$ & $5(1)$ & $3(1)$ \\
\hline $\mathrm{N}(11)$ & $14(1)$ & $17(1)$ & $24(1)$ & $0(1)$ & $3(1)$ & $3(1)$ \\
\hline $\mathrm{C}(12)$ & $15(1)$ & 19(1) & 21(1) & $-1(1)$ & $0(1)$ & $0(1)$ \\
\hline $\mathrm{C}(12 \mathrm{~A})$ & $22(1)$ & $28(1)$ & $28(1)$ & $-2(1)$ & $-5(1)$ & $-2(1)$ \\
\hline $\mathrm{C}(13)$ & 11(1) & $14(1)$ & $25(1)$ & $1(1)$ & $-1(1)$ & $-4(1)$ \\
\hline $\mathrm{N}(14)$ & $14(1)$ & $16(1)$ & 21(1) & $0(1)$ & $3(1)$ & $1(1)$ \\
\hline$C(15 A)$ & $17(1)$ & 18(1) & $32(1)$ & $3(1)$ & $-5(1)$ & $0(1)$ \\
\hline$C(15)$ & $15(1)$ & 14(1) & 21(1) & 2(1) & $1(1)$ & $1(1)$ \\
\hline$C(16)$ & $18(1)$ & $14(1)$ & $22(1)$ & $0(1)$ & $-3(1)$ & $1(1)$ \\
\hline $\mathrm{C}(17)$ & 21(1) & 21(1) & 29(1) & $-3(1)$ & $2(1)$ & $-5(1)$ \\
\hline $\mathrm{C}(18)$ & $17(1)$ & $26(1)$ & 29(1) & $-1(1)$ & $0(1)$ & $-7(1)$ \\
\hline C(19) & $29(1)$ & $33(1)$ & 23(1) & $7(1)$ & $-2(1)$ & $0(1)$ \\
\hline $\mathrm{C}\left(20^{\prime}\right)$ & $18(1)$ & $35(1)$ & 23(1) & $-5(1)$ & $2(1)$ & $1(1)$ \\
\hline $\mathrm{C}(20)$ & $30(2)$ & $26(1)$ & $37(2)$ & $-5(1)$ & $1(1)$ & $1(1)$ \\
\hline $\mathrm{C}(21)$ & $16(1)$ & $36(2)$ & $36(2)$ & $-7(1)$ & $1(1)$ & $0(1)$ \\
\hline C(22") & $39(2)$ & $46(2)$ & $30(2)$ & $-6(1)$ & $-4(1)$ & $0(2)$ \\
\hline $\mathrm{C}\left(22^{\prime}\right)$ & $40(2)$ & $38(2)$ & $53(2)$ & $3(2)$ & $-19(2)$ & $-19(2)$ \\
\hline $\mathrm{C}(22)$ & 19(1) & $50(2)$ & $41(2)$ & $-7(1)$ & $-13(1)$ & $-1(1)$ \\
\hline $\mathrm{C}(23)$ & $29(1)$ & $20(1)$ & $31(1)$ & $1(1)$ & $7(1)$ & $9(1)$ \\
\hline $\mathrm{C}\left(24^{\prime}\right)$ & $16(1)$ & $27(1)$ & $45(2)$ & $3(1)$ & $-6(1)$ & $-2(1)$ \\
\hline$C(24)$ & $34(2)$ & $32(1)$ & $31(2)$ & $5(1)$ & $-10(1)$ & $-9(1)$ \\
\hline$C(25)$ & $38(2)$ & $30(2)$ & $68(3)$ & $-17(2)$ & $27(2)$ & $-10(1)$ \\
\hline
\end{tabular}


Table S5. Hydrogen coordinates $\left(x 10^{4}\right)$ and isotropic displacement parameters $\left(\AA^{2} \times 10^{3}\right)$ for 3 .

\begin{tabular}{|c|c|c|c|c|}
\hline & $\mathrm{x}$ & $\mathrm{y}$ & $\mathrm{z}$ & $\mathrm{U}(\mathrm{eq})$ \\
\hline $\mathrm{H}(4 \mathrm{O})$ & $4620(20)$ & $-390(20)$ & $3080(20)$ & $54(10)$ \\
\hline $\mathrm{H}(3)$ & $7865(19)$ & $5530(16)$ & $517(15)$ & $22(6)$ \\
\hline $\mathrm{H}(5)$ & $9630(20)$ & $5065(16)$ & $3491(17)$ & $36(7)$ \\
\hline $\mathrm{H}(6)$ & $10950(20)$ & $4472(16)$ & 4394(18) & $32(7)$ \\
\hline $\mathrm{H}(7)$ & $12510(20)$ & $4090(18)$ & $3828(18)$ & $38(8)$ \\
\hline $\mathrm{H}(8)$ & $12910(20)$ & $4233(16)$ & $2381(15)$ & $25(7)$ \\
\hline $\mathrm{H}(11)$ & $6750(20)$ & $4516(18)$ & $639(16)$ & $30(7)$ \\
\hline $\mathrm{H}(12)$ & 7296(17) & $2783(15)$ & $565(14)$ & $13(6)$ \\
\hline $\mathrm{H}(15 \mathrm{~A})$ & $8255(17)$ & $1600(15)$ & $3360(15)$ & $17(6)$ \\
\hline $\mathrm{H}(15)$ & $6637(15)$ & $2831(14)$ & $3208(13)$ & $7(5)$ \\
\hline $\mathrm{H}(16)$ & $6409(17)$ & $987(16)$ & $2840(15)$ & $20(6)$ \\
\hline $\mathrm{H}(19 \mathrm{C})$ & $8210(20)$ & $5710(20)$ & $-977(19)$ & 49(9) \\
\hline $\mathrm{H}(19 \mathrm{~B})$ & $9310(30)$ & $5510(20)$ & $-1350(20)$ & $65(10)$ \\
\hline $\mathrm{H}(19 \mathrm{~A})$ & $8570(20)$ & $4720(20)$ & $-1045(17)$ & $37(7)$ \\
\hline $\mathrm{H}(20 \mathrm{E})$ & $8113(18)$ & $4555(16)$ & $2332(14)$ & $18(6)$ \\
\hline $\mathrm{H}(20 \mathrm{D})$ & $8304(17)$ & $5533(15)$ & $2775(16)$ & $20(6)$ \\
\hline $\mathrm{H}(20 \mathrm{~F})$ & $7440(20)$ & $5360(15)$ & 2090(16) & $22(6)$ \\
\hline $\mathrm{H}(20 \mathrm{C})$ & 9191(18) & $6830(15)$ & 2019(16) & $20(6)$ \\
\hline $\mathrm{H}(20 \mathrm{~B})$ & $9219(19)$ & $6797(16)$ & $1025(17)$ & $23(7)$ \\
\hline $\mathrm{H}(20 \mathrm{~A})$ & $8110(20)$ & $6797(18)$ & $1473(18)$ & $43(8)$ \\
\hline $\mathrm{H}(21 \mathrm{~B})$ & $13010(30)$ & $4910(20)$ & $970(20)$ & $59(9)$ \\
\hline $\mathrm{H}(21 \mathrm{~A})$ & $12260(20)$ & $4740(20)$ & $110(20)$ & 49(9) \\
\hline $\mathrm{H}(21 \mathrm{C})$ & $12550(20)$ & $3940(20)$ & $740(20)$ & $55(9)$ \\
\hline $\mathrm{H}(22 \mathrm{~L})$ & $6630(20)$ & $2370(20)$ & $-825(19)$ & $52(9)$ \\
\hline $\mathrm{H}(22 \mathrm{~K})$ & $6290(20)$ & 3393(19) & $-929(17)$ & $33(7)$ \\
\hline $\mathrm{H}(22 \mathrm{H})$ & $5460(30)$ & $2670(20)$ & $-1080(20)$ & $61(10)$ \\
\hline $\mathrm{H}(22 \mathrm{~F})$ & $4910(20)$ & 1798(19) & $220(20)$ & $45(8)$ \\
\hline $\mathrm{H}(22 \mathrm{E})$ & $5230(20)$ & $2050(20)$ & $1240(20)$ & $60(9)$ \\
\hline $\mathrm{H}(22 \mathrm{D})$ & $6000(30)$ & $1530(20)$ & $500(20)$ & $58(10)$ \\
\hline $\mathrm{H}(22 \mathrm{~B})$ & $4360(20)$ & $3356(16)$ & $37(17)$ & $31(7)$ \\
\hline $\mathrm{H}(22 \mathrm{~A})$ & $4670(20)$ & $3600(20)$ & $1040(20)$ & $57(9)$ \\
\hline $\mathrm{H}(22 \mathrm{C})$ & $5140(20)$ & $4170(20)$ & 194(19) & $45(8)$ \\
\hline $\mathrm{H}(23 \mathrm{C})$ & $8367(19)$ & $2098(17)$ & $1329(16)$ & $26(6)$ \\
\hline $\mathrm{H}(23 \mathrm{~A})$ & $8150(20)$ & $1390(20)$ & $2110(20)$ & $50(8)$ \\
\hline $\mathrm{H}(23 \mathrm{~A})$ & $7470(30)$ & $1360(20)$ & $1330(20)$ & $60(10)$ \\
\hline $\mathrm{H}(24 \mathrm{~F})$ & $8390(20)$ & $3528(19)$ & $3499(16)$ & $34(7)$ \\
\hline $\mathrm{H}(24 \mathrm{E})$ & 8890(20) & $3017(17)$ & $2720(19)$ & $36(7)$ \\
\hline $\mathrm{H}(24 \mathrm{D})$ & $9330(20)$ & 2854(17) & $3666(17)$ & $34(7)$ \\
\hline $\mathrm{H}(24 \mathrm{C})$ & $7150(20)$ & $1672(18)$ & $4613(18)$ & $41(8)$ \\
\hline $\mathrm{H}(24 \mathrm{~B})$ & $7420(20)$ & $2690(20)$ & $4680(20)$ & $48(9)$ \\
\hline $\mathrm{H}(24 \mathrm{~A})$ & $8340(30)$ & 1970(20) & $4790(20)$ & $56(9)$ \\
\hline $\mathrm{H}(25 \mathrm{C})$ & $5260(30)$ & $2830(20)$ & $3850(20)$ & $57(9)$ \\
\hline $\mathrm{H}(25 \mathrm{~B})$ & 4180(30) & $2340(20)$ & $3920(20)$ & $65(11)$ \\
\hline $\mathrm{H}(25 \mathrm{~A})$ & $5050(40)$ & $2180(30)$ & $4600(30)$ & $130(20)$ \\
\hline
\end{tabular}




\section{References and Notes.}

${ }^{1}$ Sperry, S.; Valeriote, F. A.; Corbett, T. H.; Crews, P. J. Nat. Prod. 1998, 61, 241-247.

2 Thale, Z.; Johnson, T.; Tenney, K.; Wenzel, P. J.; Lobkovsky, E.; Clardy, J.; Media, J.; Pietraszkiewicz, H.; Valeriote, F. A.; Crews, P. J. Org. Chem. 2002, 67, 9384-9391.

3 SMART \& SAINT Software Reference manuals, version 5.042; Bruker Analytical X-ray Systems. Madison WI, 1998.

${ }^{4}$ Sheldrick G.M. SADABS, a software for empirical absorption correction; University of Gottingen, Germany, 2000.

5 Sheldrick G.M. SHELXTL Reference manuals, version 5.1; Bruker Analytical X-ray Systems. Madison WI, 1997

6 Tinley, T. L.; Randall-Hlubek, D. A.; Leal, R. M.; Jackson, E. M.; Cessac, J. W.; Quada, J. C., Jr.; Hemscheidt, T. K.; Mooberry, S. L. Cancer Res. 2003, 63, 3211-3220.

${ }^{7}$ Ratnayake, A. S.; Yoshida, W. Y.; Mooberry, S. L.; Hemscheidt, T. Org. Lett. 2001, 3, 3479-3481. 